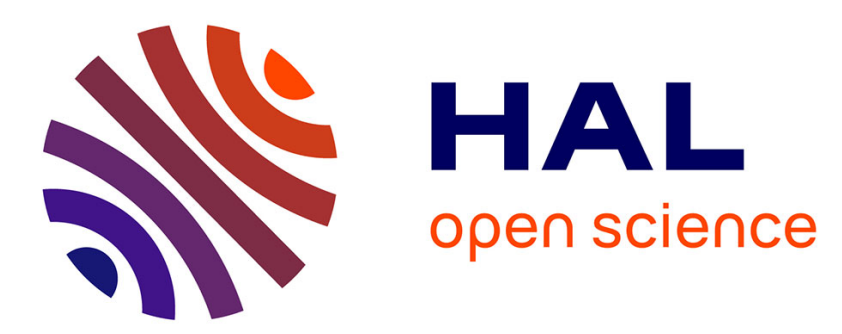

\title{
Observation de super-réseaux CdTe-HgTe par microscopie électronique en transmission
}

L. Di Cioccio, A. Million, J.P. Gailliard, M. Dupuy

\section{To cite this version:}

L. Di Cioccio, A. Million, J.P. Gailliard, M. Dupuy. Observation de super-réseaux CdTe-HgTe par microscopie électronique en transmission. Revue de Physique Appliquée, 1987, 22 (6), pp.465-468. 10.1051/rphysap:01987002206046500 . jpa-00245561

\section{HAL Id: jpa-00245561 https://hal.science/jpa-00245561}

Submitted on 1 Jan 1987

HAL is a multi-disciplinary open access archive for the deposit and dissemination of scientific research documents, whether they are published or not. The documents may come from teaching and research institutions in France or abroad, or from public or private research centers.
L'archive ouverte pluridisciplinaire HAL, est destinée au dépôt et à la diffusion de documents scientifiques de niveau recherche, publiés ou non, émanant des établissements d'enseignement et de recherche français ou étrangers, des laboratoires publics ou privés. 


\title{
Observation de super-réseaux CdTe-HgTe par microscopie électronique en transmission
}

\author{
L. Di Cioccio, A. Million, J. P. Gailliard et M. Dupuy \\ Commissariat à l'Energie Atomique, Institut de Recherche et Développement Industriel, \\ Laboratoire d'Electronique et de Technologie de l'Informatique, BP 85 X, CEN, \\ 38041 Grenoble, France
}

(Reçu le 17 novembre 1986, accepté le 13 février 1987)

Résumé. - La microscopie électronique en transmission est utilisée pour la visualisation de super-réseaux CdTe-HgTe. Des défauts tels que défauts d'empilement, ségrégation d'impuretés et dislocations sont observés.

Abstract. - High resolution transmission electron microscopy is used for CdTe-HgTe superlattices observation. Defects, such as stacking faults, impurity segregation and dislocations are observed.

\section{Introduction.}

La réalisation de super-réseaux III-V, obtenus par épitaxie par jet moléculaire (EJM) du type GaAsGaAlAs par exemple, a fait l'objet de nombreuses publications [1]. L'utilisation de l'EJM dans les semi-conducteurs II-VI a été beaucoup plus tardive [2]. Dans cette dernière famille, le système CdTeHgTe présente l'avantage d'un accord de maille quasi parfait entre les deux composés $(a=0,648 \mathrm{~nm}$ pour CdTe contre $a=0,646 \mathrm{~nm}$ pour $\mathrm{HgTe}$ ). L'observation par microscopie électronique en transmission (MET) de coupes transversales minces de tels super-réseaux est récente [3]. L'utilisation du microscope en haute résolution (METHR), apporte de nombreuses informations sur les interfaces, en particulier sur les défauts cristallins en relation avec ces interfaces. Nous nous proposons de présenter nos résultats relatifs à l'observation de défauts par METHR dans des super-réseaux CdTe-HgTe.

\section{Méthodes expérimentales.}

2.1 EPITAXIE PAR JET MOLÉCULAIRE. - Les superréseaux CdTe-HgTe ont été épitaxiés sur un substrat CdTe d'orientation (111) A à une température de $468 \mathrm{~K}$. La préparation des surfaces a été décrite par ailleurs [2]. La croissance des couches de CdTe est effectuée en utilisant une seule cellule contenant du CdTe, l'évaporation de ce composé étant congruente [4]. Pour HgTe, deux cellules (mercure et tellure) ont été utilisées. Les vitesses de dépôt sont de $0,2 \mathrm{~nm} \cdot \mathrm{s}^{-1}$. Les super-réseaux étudiés sont pour la plupart constitués d'une centaine de périodes, d'épaisseur d'environ $8 \mathrm{~nm}$ pour les deux composants. La première couche épitaxiée est HgTe. L'épaisseur critique de couche individuelle à partir de laquelle l'épitaxie n'est plus cohérente entre ces deux matériaux, qui ont un désaccord de paramètre de maille $\frac{\Delta a}{a}$ égal à $0,3 \%$, est d'environ $360 \mathrm{~nm}$ [5]. L'épitaxie s'effectuant sur substrat CdTe, les couches de $\mathrm{HgTe}$ vont donc être contraintes. La dernière couche du super-réseau est CdTe, ce qui permet d'encapsuler le $\mathrm{HgTe}$ et d'éviter toute évaporation de mercure au moment de l'arrêt de la croissance.

2.2 MiCROSCOPIE ÉlECTRONIQUE EN TRANSMISSION. - Les échantillons sont préparés par amincissement de coupes transversales à l'amincisseur ionique (ions d'argon) suivant une méthode déjà décrite [6]. Dans le but de minimiser les défauts d'irradiation et le mixage du super-réseau pendant l'amincissement, la tension d'accélération est maintenue à $2 \mathrm{kV}$ sous $25 \mu \mathrm{A}$ de débit sous un angle d'attaque de $15^{\circ}$. Le plan d'observation est (110) perpendiculaire au plan de croissance (111). Le microscope utilisé est un JEM $200 \mathrm{CX}$ à filament $\mathrm{LaB}_{6}$, platine « topentry » et lentille objective de faible aberration sphérique $(\mathrm{Cs}=1,05 \mathrm{~mm})$. A $200 \mathrm{kV}$, les échantillons ne subissent pas de défauts d'irradiation, cepen- 
dant lors de l'observation de zones très minces $(\leqslant 10 \mathrm{~nm})$ une évaporation du mercure, par échauffement local sous le faisceau, peut se produire.

\section{Résultats.}

3.1 SUPER-RÉSEAU. - La figure 1 représente un super-réseau CdTe-HgTe en haute résolution où le CdTe correspond aux bandes claires. On peut vérifier qu'en moyenne, on réalise 9 couches de CdTe pour 18 couches de $\mathrm{HgTe}$ (environ 3,4 et $6,7 \mathrm{~nm}$ respectivement). On constate qu'à l'échelle de la distance interatomique, les interfaces sont rendues irrégulières par la présence de marches. Il est possible aussi que, malgré les précautions prises lors de l'amincissement (débit et énergie des ions faibles), une interdiffusion des deux composés ait eu lieu.

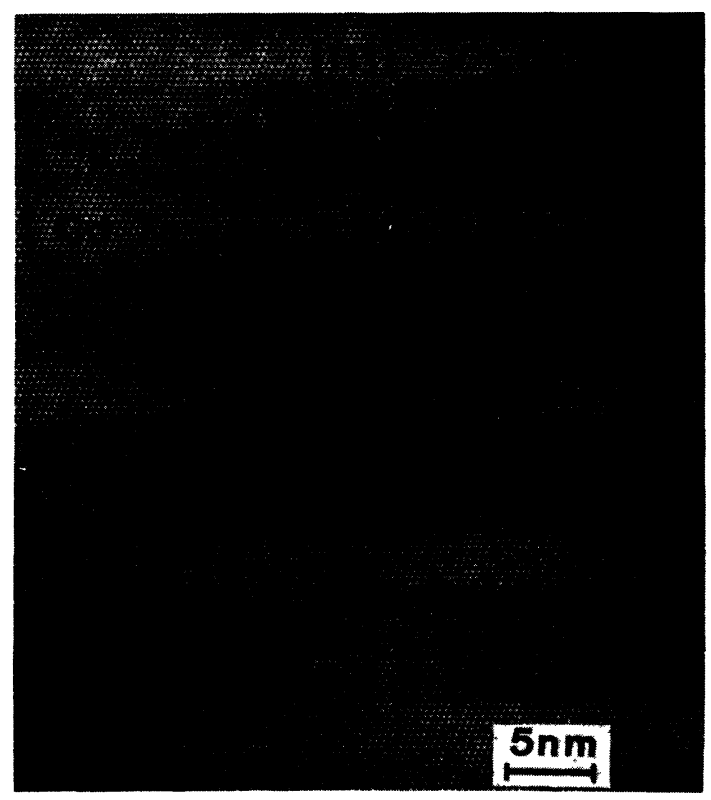

Fig. 1. - Super-réseau CdTe-HgTe observé en microscopie électronique haute résolution. Les épaisseurs relatives des couches sont respectivement 3,4 et $6,7 \mathrm{~nm}$.

[High resolution transmission electron microscopy of $\mathrm{CdTe}-\mathrm{HgTe}$ superlattice. The corresponding layers are 3.4 and $6.7 \mathrm{~nm}$ thick.]

3.2 DÉFAUT A L'INTERFACE SUBSTRAT SUPERRÉSEAU. - Une mauvaise préparation de la surface du substrat peut provoquer un désordre très important dans les premières couches du super-réseau (Fig. 2) provoqué par la présence de précipités à l'interface. Toutefois, après 5-7 périodes, le superréseau est régulier. A plus fort grossissement (Fig. 3) on remarque la présence de défauts d'empilement à l'interface mais qui ne conduisent pas à une croissance en relation de macle. Les contrastes noirs (i) ont été attribués à la présence d'impuretés. Dans ce cas on note aussi la présence de dislocations à

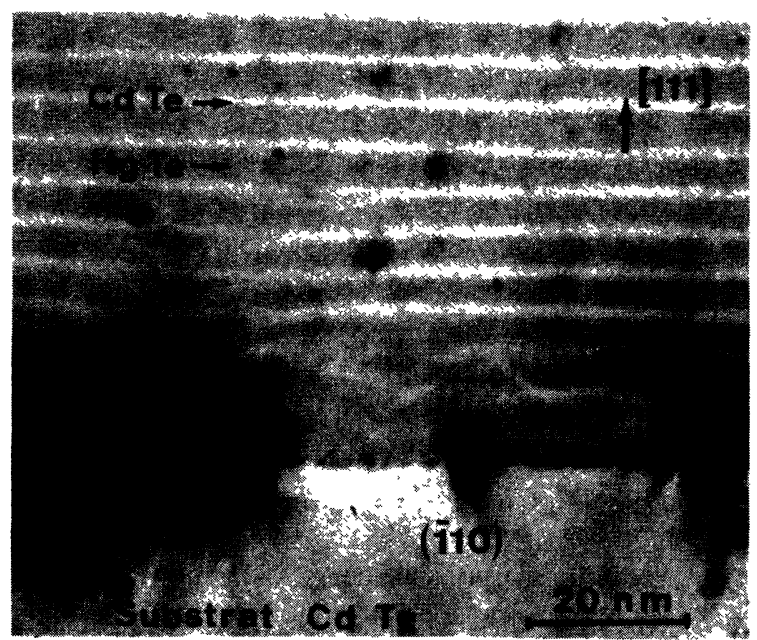

Fig. 2. - Premières couches du super-réseau sur un substrat mal préparé.

[An example of the first layers grown on a badly prepared substrate.]

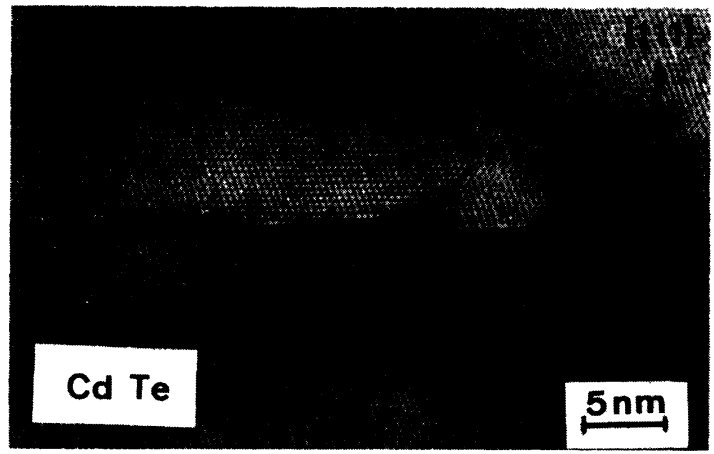

Fig. 3. - Défauts d'empilement, ségrégation d'impuretés (i) et dislocation (D) à l'interface substrat super-réseau. Même échantillon que celui de la figure 2 .

[Stacking faults, impurity segregation (i) and dislocation (D) at the substrate-superlattice interface. Same specimen as figure 2.]

$60^{\circ}$ (D), la plupart du temps associées à des impuretés et dont nous reparlerons plus loin. Enfin, même pour les meilleures préparations de surface, nous avons toujours obtenu des contrastes dus aux impuretés à l'interface substrat-première couche, qui décorent celle-ci (Fig. 4). La localisation précise de l'interface est délicate dès lors que l'échantillon est parfaitement orienté suivant (110). Il est alors nécessaire de procéder à une légère désorientation pour exciter une diffraction de type 002 dont l'intensité est très différente dans CdTe et $\mathrm{HgTe}$ (cas de la Fig. 1).

3.3 Dislocations. - Dans les couches de bonnes qualités, les dislocations sont très rares et donc très difficiles à observer surtout en haute résolution. 


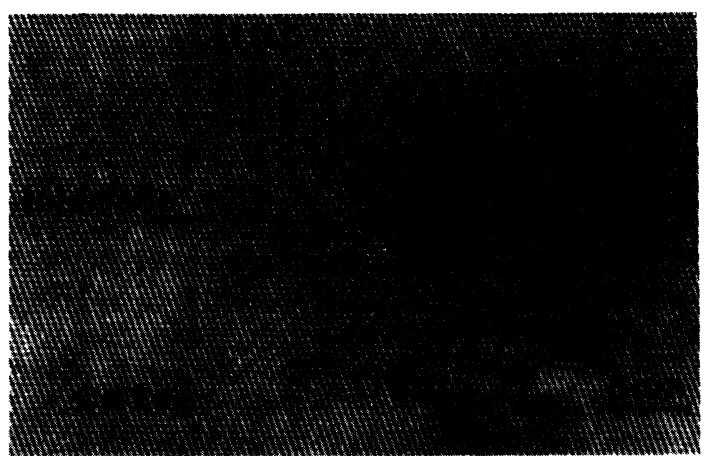

Fig. 4. - Impuretés résiduelles à l'interface CdTe superréseau. Cas d'une préparation correcte de la surface du substrat.

[Residual impurities at the CdTe-superlattice interface. Case of a well prepared substrate surface.]

Toutefois, nous avons pu observer quelques dislocations à $60^{\circ}$ à l'interface substrat-première couche et une dislocation de Lomer dans le super-réseau.

3.3.1 Dislocation à $60^{\circ}$. - La figure 5 représente une dislocation à $60^{\circ}$ située très près de l'interface substrat CdTe-première couche de $\mathrm{HgTe}$ et dans le $\mathrm{HgTe}$. Cette dislocation est associée à la présence d'impuretés. Elle a comme plan de glissement (111) et a pu être générée à partir de la surface de l'échantillon au cours des premiers stades de

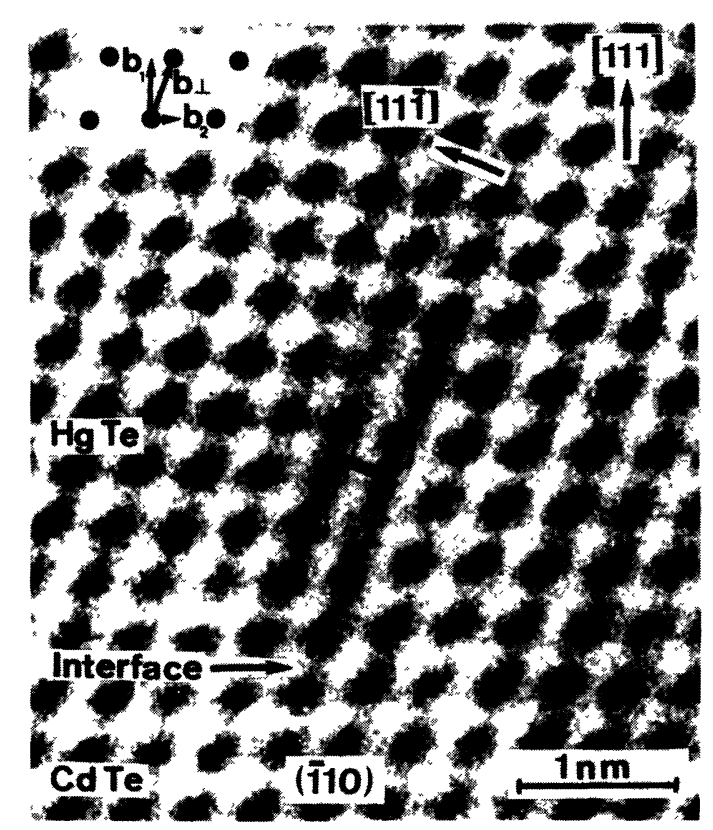

Fig. 5. - Dislocation à $60^{\circ}$ au voisinage de l'interface substrat CdTe-première couche de $\mathrm{HgTe}$. Les composantes coin sont $b_{1}=1 / 3[111]$ et $b_{2}=1 / 12[112]$.

$\left[60^{\circ}\right.$ dislocation in the vicinity of the CdTe substrate-first HgTe layer interface. The edge components are $b_{1}=$ $1 / 3[111]$ and $b_{2}=1 / 12$ [112].] l'épitaxie et glisser jusqu'au voisinage de l'interface. Elle pourrait ainsi relaxer des contraintes générées par les impuretés à l'interface. Son vecteur de Burgers est $b=1 / 2$ [011]. Sur la figure 5 sa composante coin $b_{\perp}=1 / 4[\overline{1} 12]$ est décomposée en deux composantes $b=1 / 3[111]$ et $b=1 / 12[\overline{1} 12]$ respectivement perpendiculaire et parallèle à l'interface. Cette dislocation est peu ou pas dissociée et est tout à fait semblable à celles observées par Hutchison [7] dans du silicium déformé.

3.3.2 Dislocation de Lomer. - Nous avons observé une dislocation de Lomer $b=1 / 2$ [110] située dans le super-réseau, dans une couche de $\mathrm{HgTe}$, décorée elle aussi par des impuretés. Cette dislocation dont l'image est représentée sur la figure 6 a un cœur compact (absence de dissociation au cœur, contrairement au modèle de cœur étendu de Lomer-Cottrell [8]). Elle est identique à celle obtenue par Bourret [9] dans le germanium. En particulier, l'image est dissymétrique près du cœur : par exemple, les canaux marqués d'une flèche sont dissymétriques en position et intensité.

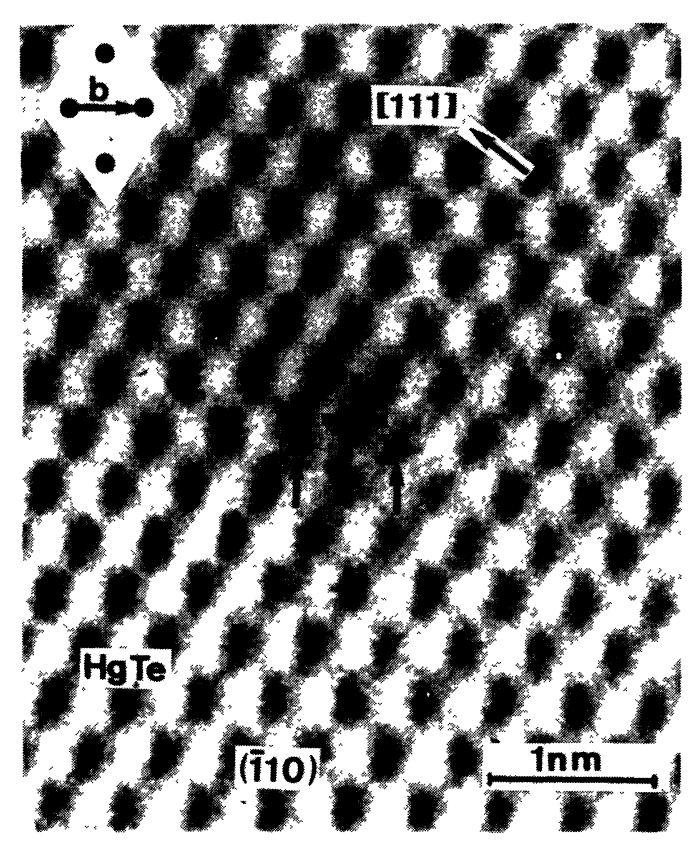

Fig. 6. - Dislocation de Lomer $(b=1 / 2[110])$ dans une couche de $\mathrm{HgTe}$ de direction de croissance [111]. L'image est dissymétrique près du cœur (voir flèches). Les points noirs correspondent aux canaux.

[Lomer dislocation $(b=1 / 2[110])$ in a HgTe layer of [111] growth direction. The core image is asymmetric (see arrows). Black dots correspond to the tunnels.]

Il est connu que lorsqu'une épitaxie est réalisée sur un substrat de CdTe (111), la qualité de cette épitaxie est a priori fort différente suivant la face (cadmium ou tellure) sur laquelle elle est réalisée. Si 
l'on admet, à partir des publications très contradictoires [10] sur la détermination du type de face, que c'est la face cadmium qui est la meilleure face pour l'épitaxie, on voit que l'on peut identifier la nature des atomes dans le cœur de la dislocation. Une telle représentation est donnée sur la figure 7 a pour le modèle symétrique de Hornstra [11] et sur la figure $7 \mathrm{~b}$ pour le modèle dissymétrique de Bourret [9], la figure 7c schématisant le substrat de départ avec une face cadmium. Par exemple on note que dans le modèle de Hornstra, les cycles à 7 et 5 colonnes près du cœur ont en commun les colonnes de tellure c'est-à-dire celles composées des plus petits atomes. De même, dans le modèle de Bourret, le cycle à 5 colonnes en comporte 3 de tellure pour 2 de mercure. Ainsi par cette méthode, nous montrons qu'il est possible de positionner les colonnes atomiques de différente nature chimique dans un semiconducteur composé bien que la résolution du microscope employé soit supérieure à la distance des colonnes atomiques mises en jeu $(0,162 \mathrm{~nm}$ entre les colonnes de $\mathrm{Hg}$ et $\mathrm{Te}$ dans $\mathrm{HgTe}$ ).

\section{Conclusion.}

L'observation en METHR de super-réseau CdTeHgTe permet l'identification des différents défauts d'interface (précipités, défauts d'empilement, dislocations). En outre nous avons montré que la connaissance de la nature chimique de la face de départ lors d'une épitaxie sur un plan (11i) permet l'identification chimique des atomes dans le cœur des dislocations. Cette méthode pourrait être utilisée, d'une

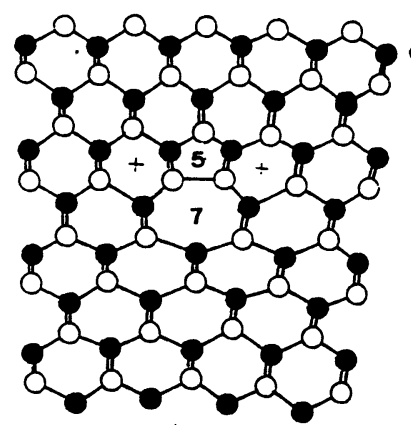

(a)

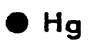

$\mathrm{OTe}$

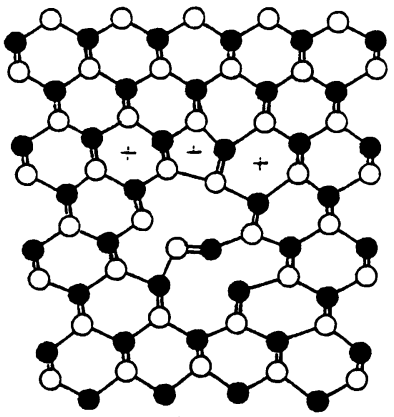

(b)

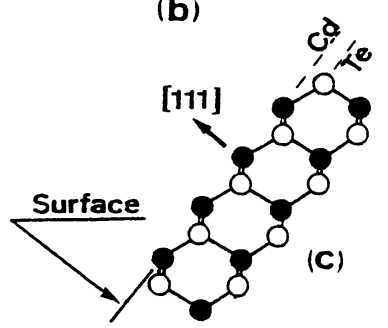

Fig. 7. - Modèle symétrique de Hornstra (a) et dissymétrique de Bourret (b) de la dislocation de Lomer. Les colonnes de $\mathrm{Hg}$ et $\mathrm{Te}$ ont été positionnées en supposant une face cadmium comme face de départ de l'épitaxie sur substrat CdTe (c). La direction de croissance est [111].

[Symmetrical Hornstra's model (a) and asymmetric Bourret's model (b) for the Lomer dislocation. $\mathrm{Hg}$ and $\mathrm{Te}$ columns have been positionned, assuming a cadmium surface for the CdTe substrate on which the epitaxial layers are grown (c). The growth direction is [111].]

façon générale, pour une étude détaillée des défauts dans les semi-conducteurs composés.

Ce travail a été financé par la DRET.

\section{Bibliographie}

[1] Chang, L. L., J. Vac. Sci. Technol. B 1 (2) (1983) 120.

[2] Faurie, J. P., Million, A. and Piaguet, J., Appl. Phys. Lett. 41 (1982) 713.

[3] Harris, K. A., Hwang, S., Blanks, D. K., Cook, J. W., Schetzin, J. F., OtSuKa, N., Baukus, J. P. and Hunter, A. T., Appl. Phys. Lett. 48 (1986) 396.

[4] Faurie, J. P., Million, A., J. Crystal Growth 54 (1981) 577.

[5] Fontaine, C., Thèse Univ. Scientifique et Médicale Grenoble (1986).
[6] Dupuy, M., J. Microsc. Spectrosc. Electron. 9 (1984) 163.

[7] Hutchison, J. L., Anstis, G. R. and Pirouz, Microscopy of semiconducting materials, Oxford, 1983, Inst. Phys. Conf. $n^{\circ} 67$, The Institute of Physics, Londres, 1983, page 21.

[8] Cottrell, A. H., Philos. Mag. 43 (1952) 645.

[9] Bourret, A., Desseaux, J. and Renault, A., Philos. Mag. A 45 (1982) 1.

[10] Fewster, P. F., Whiffin, P. A. C., J. Appl. Phys. 54 (8) (1983) 4668.

[11] Hornstra, J., Phys. Chem. Solids 5 (1958) 129. 